\title{
Imaginer, monter : la mémoire inachevée d'Auschwitz selon Georges Didi-Huberman
}

\author{
Adina BALINT-BABOS, Université de Winnipeg
}

\section{Introduction}

L'histoire contemporaine et la mémoire qu'elle se donne pour tâche de représenter depuis l'épreuve des camps de la Deuxième Guerre mondiale, sont marquées par un bouleversement du principe de narration. Apparaissent d'autres modes de transmission parmi lesquels le témoignage et les images, dont les conditions de lisibilité, l'analyse et la valeur de connaissance sont à considérer suivant les discours qui les véhiculent. La complexité du travail se noue ainsi entre les discours d'une mémoire officielle et l'élaboration subjective du passé de la guerre, de cette crise de l'histoire qui ne cesse de revenir sous la plume des écrivains de deuxième et troisième générations après la Shoah. Autant dire que la mémoire des camps tend à relever du complexe, du symptôme, d'une affaire de transmission mal réglée, ce qui nous conduit précisément à réfléchir sur les modalités discursives et politiques de ces retours.

La mémoire de la Shoah, qui reste à penser dans son actualité, s'est constituée à partir de deux types de traces : la parole - soit la littérature, le témoignage littéraire et historique - et l'image, les images et les documents qui sont parvenus jusqu'à nous. Dans ce processus, la réflexion esthétique et les possibilités ouvertes par l'art (Christian Boltanski, par exemple) se sont imposées comme espaces déterminants dans la constitution critique d'une mémoire de la Shoah. Cependant, les images et les paroles appelées à ce sujet supposent la mise en lumière d'une mécanique de mise à mort, qui visait d'emblée la destruction de toute trace de corps et la suspension de toute pensée du terrible, du désastre. Malgré tout, les paroles nous informent, les images nous regardent; paroles et images pénètrent jusque dans nos rêves.

L'imagination (principe de surgissement des formes) et le montage (principe d'écriture) vont de pair dans cet effort de mémoire qui nous questionne sur les limites de notre humanité, sur l'ambivalence de nos regards et sur les brouillages inconscients de ces images, entre le réel du corps et le réel de l'histoire.

En travaillant l'imagination et le montage, Georges Didi-Huberman a dégagé de nouvelles voies dans ce processus de mémoire et de remémoration : pour lui, l'imagination n'est pas séparée du réel de l'histoire et le montage apparaît dans sa valeur de connaissance, de nomination, 
d'énonciation. Et tout cela autour d'un objet singulier : la série de quatre photographies prises en 1944 à Auschwitz, et qu'il a accepté de regarder, de nommer, de décrire, de nous rendre lisibles.

\section{« Pour savoir, il faut s'imaginer »}

Ainsi commence Images malgré tout, l'essai de Didi-Huberman publié en 2003. Cette première proposition, qui mobilise les images au cœur d'une réflexion politique sur la question des camps, a suscité de multiples controverses, à partir des problèmes complexes d'ordre éthique, politique et esthétique convoqués par les images elles-mêmes en tant que représentations, mémoire, connaissance historique. C'est contre la question de l'irreprésentable défendue par Claude Lanzmann dans un refus de la valeur d'archive des images de la Shoah ${ }^{1}$, que DidiHuberman défend la validité documentaire et historique de ces images, la nécessité de les rappeler et de les inscrire au champ de l'expérience. Il s'agit de rendre à ces images leur lisibilité, mais aussi de rendre compte de leur difficile temporalité. Et de nous restituer la possibilité d'une « expérience du regard » (21).

Si aujourd'hui la représentation de la Shoah est mise en question parce qu'elle est tenue pour impossible ou rendue interdite - «par le rabattement, comme l'explique Jacques Rancière, des problèmes de réglage de la distance représentative en problèmes d'impossibilité de la représentation » («S'il y a »,81), dans un débat où l'esthétique du sublime prétend invalider toute analyse sur « l'archive photographique » (43) - la question de l'imagination est posée positivement par Didi-Huberman. Elle peut évidemment être traitée sur le mode de l'impossible imaginable, mais elle apparaît également comme « faculté subjective d'un regard possible » (Images, 55).

L'imagination avec le montage : l'imagination est d'emblée un montage, de par les images qu'elle associe, qu'elle laisse ressurgir, remonter. Une part du travail de l'imagination est ainsi consciente, liée à l'organisation de la pensée. Une autre part en est l'inconscient, le symptôme, le condensé, si bien que l'imagination agence les images suivant des relations, des correspondances, des rythmes entre liaison et déliaison, ressemblance et dissemblance, synthèse et déconstruction qu'il est question finalement d'arrêter et de monter, ne serait-ce que pour les exposer, les interpréter. «Le montage serait le moment éthique et esthétique de l'imagination » (98) parce qu'il est travail sur la forme et mise en forme. Il est principe de l'écriture des images. 


\section{« L'historien-interprète des rêves »}

Didi-Huberman a regardé les images de la Shoah d'abord en « historien-interprète des rêves » (33) - comme l'ont été avant lui Walter Benjamin et Aby Warburg - c'est dire en historien qui s'autorise d'imaginer; qui laisse les images se déplier les unes après les autres, se mêler entre deux sommeils, se heurter, passer de la fiction fantasmatique à une organisation raisonnée où ces différents registres de l'imagination peuvent s'articuler et produire du sens :

Imaginer, n'est-ce pas d'abord se mettre à l'écoute d'images interposées en un lieu psychique où l'arbitraire des associations «subjectives » fait lever une structure «objective» de correspondances ou, du moins, une certaine orientation de l'existence même ? (Didi-Huberman, « La muse », 40)

Or, ce travail d' « élaboration » - comme on dit en psychanalyse, « on élabore » pour dire qu'on établit des associations, pour dire aussi que ces associations aient un sens -, ce travail demande du temps. Le temps pour ces images de nous regarder, le temps pour nous d'être touchés, de les identifier ; de les oublier peut-être. Et enfin, de les retrouver après coup, plus tard, dans un nouveau montage, un nouvel agencement.

L'expérience du regard de Didi-Huberman sur les quatre photographies prises à Auschwitz nous engage à penser qu'il s'agirait selon lui de trouver, par le montage, « le principe de mise en forme de l'imagination » (Images, 77). Si, comme le pense Benjamin, l'imagination opère par « déformation », par « dissolution des formes » ${ }^{2}$, si elle fait avancer les images dans un flux, alors le travail de montage est nécessaire à l'élaboration critique de l'imagination, à son exposition. Plus fondamentalement, le montage et l'imagination sont immanents à l'image elle-même, en ce que l'image ne prend sens pour Didi-Huberman que dans le contact avec une autre. L'image est donc à montrer et à monter, et c'est dans les relations qu'elle établit avec les autres que se dessine sa fonction discursive, éthique, esthétique, politique et son historicité.

Il est intéressant de noter qu'une grande partie des travaux de Didi-Huberman engage une réflexion ininterrompue et continuellement inachevée sur l'histoire à partir d'une « connaissance par le montage » (64). La question historique de la Shoah précisément se trouve saisie par le débat philosophique et littéraire entre ces deux extrêmes : la défaite de la spéculation théorique ou documentaire et un excès d'images, ou des images en excès. C'est dans ce cadre que l'art, et parfois des récits-photos, sont apparus comme lieux d'asile de ces images, de ces témoignages à monter, et comme espaces de constitution d'une mémoire commune. 


\section{Écorces - l'inachevé d'un récit-photo}

Il arrive qu'un livre de quatre-vingts pages, qui n'est pas le livre d'un témoin d'Auschwitz, un livre qui se lit comme un long poème en prose, produise un effet aussi fort que des ouvrages documentés, érudits, sur la Deuxième Guerre mondiale, ou des témoignages de ceux qui sont revenus de «là-bas » (Écorces, 9). Écorces, le livre bref de Didi-Huberman, publié aux Éditions de Minuit en 2011, pourrait être rangé aux côtés des essais bien connus, comme par exemple $S i$ c'était un homme de Primo Levi ou Être sans destin de Imre Kertész.

Écorces est le récit d'un voyage, d'une déambulation, que Didi-Huberman a fait en Pologne, à Auschwitz-Birkenau en juin 2010. C'est une tentative d'interroger le sol du Crématoire V et les arbres, les bouleaux, qu'il fallait photographier pour voir ce qui survit aujourd'hui de la mémoire de la Shoah, ce qui pourrait mettre en œuvre le désir de ne pas rester au deuil crispé du lieu de la mise à mort. De ce voyage, Didi-Huberman a rapporté trois petits morceaux d'écorce, prélevés sur un bouleau. On sait que le mot Birken signifie bouleaux en allemand, la terminaison au désignant la prairie où poussent les bouleaux, mais cette terminaison est aussi proche de l'exclamation de la douleur en allemand, $a u$.

Qu'est-ce qu'on peut attendre d'une photo d'un morceau d'écorce de bouleau dans un endroit comme Birkenau? Le bout d'écorce, donc, qui a été le déclencheur de l'écriture d'Écorces, «n'est pas la révélation de l'arbre, soutient Didi-Huberman, c'est quelque chose à partir de quoi, je peux réfléchir à l'arbre, tout en sachant que je n'aurai pas la révélation de la totalité de l'arbre » (23). C'est autour de cette question du partiel, de la production de «petites vérités » (8) en tant qu'inachèvement de l'espace mémoriel de la Shoah qui se transmet au présent - que se tisse la digression philosophique du récit Écorces.

Par ailleurs, comme nous l'avons souligné plus haut, dans l'essai Images malgré tout, DidiHuberman réfléchissait déjà à ce qui se transmet dans le présent d'un passé trouble (comme la Deuxième Guerre mondiale). Il achevait son ouvrage sur les quatre photos prises par les membres du Sonderkommando d'Auschwitz-Birkenau par ces mots : «La question des images est au cœur de ce grand trouble du temps (la Deuxième Guerre), notre «malaise dans la culture ». Il faudrait savoir regarder les images, ce dont elles sont les survivantes. Pour que l'histoire, libérée du pur passé (cet absolu, cette abstraction) nous aide à ouvrir le présent du temps » (226).

Il convient de noter que l'essai Images malgré tout analysait des photographies faites clandestinement à Birkenau, en 1944, par un déporté membre d'un Sonderkommando. Celui-ci a 
pris au moins quatre photos qui constituent le seul témoignage visuel d'une opération de gazage en train de se dérouler.

Des questions surgissent ici, directement ou en filigrane : Pourquoi vouloir témoigner par l'image ? En quoi ces images peuvent-elles être un témoignage ; témoignage de quoi ? Que peuvent-elles montrer des camps ? Ou encore, quel lien existe-t-il entre l'image et le témoignage? On retrouve dans Écorces des questions proches, même si le questionnement y est plus personnel : les photographies sont celles, récentes, de l'auteur; il est question de sa propre visite des camps, dont il avoue : «J'ai réinscrit, chemin faisant, ce lieu dans mon histoire familiale, mes grandsparents morts ici même » (42).

Dans d'autres mots, Écorces est l'exploration d'un regard et l'exploration, par le regard, de ce qui est regardé. Ce qui est regardé : ce sont des photographies, ainsi qu'un lieu, AuschwitzBirkenau. Il y a dix-neuf photographies reproduites dans le livre : photographies des bouleaux, du sol, de ce qui reste des crématoires - il n'y a aucun être humain dans ces prises de vue - comme si le but du voyage était premièrement soutenu par la nécessité de réinterroger encore les lieux, le paysage, le sol, la lumière, le ciel...

\section{Écrire l'écorce, monter l'image}

Symboliquement, l'écorce est une interface entre l'arbre et le monde, qui va servir de point de départ à la méditation-digression de l'écrivain, qui produit dans Écorces une série de courts chapitres s'ouvrant chacun par une photo en noir et blanc, prise sur place à Birkenau. « Là-bas » (9), écrit Didi-Huberman, il a eu l'impression de poser une question d'abord muette, aux bouleaux ; les bouleaux, qui dans cette région de la Pologne ont une longévité supérieure aux trente ans habituels pour cette espèce, et qui sont donc à certains égards les seuls témoins et les seuls survivants de Birkenau, bouleaux situés à portée immédiate des chambres à gaz.

La question est d'abord muette, et Didi-Huberman souligne avec justesse le caractère inimaginable de ce qui s'est passé là où il se trouve. Mais il a conscience aussi que cette « impasse de l'imagination » (34) faisait partie des forces stratégiques des SS et qu'il faut donc tenter d'en sortir ; origine peut-être, point déclencheur des photos, sur place ; puis du texte, venu un peu par surprise, un peu plus tard, revenu à Paris.

Sous deux angles au moins, Écorces a trait à l'histoire personnelle de son auteur. Il y évoque des photos prises en 1944, dans des conditions de danger extrême, par des membres d'un 
Sonderkommando devant le Crématoire V. Les photographies faites à Birkenau en 1944 - prises à l'aveugle, dans l'urgence, grâce à un appareil dissimulé dans un seau -, dont il est question dans Écorces, trouvent leur sens et leur valeur dans ce qu'elles montrent leur référent, mais aussi dans le fait qu'elles ont été prises ; et dans ce qu'elles font transparaître du contexte qui les rend possibles, même celle qui, des quatre, peut sembler ratée. Contrairement aux trois premières prises de vue, cette dernière ne montre personne mais, de manière floue, des feuillages. Pourtant, si nous $[\ldots]$ acceptons de la regarder, cette photographie « ratée », « abstraite » ou « désorientée », témoigne de quelque chose qui demeure essentiel : elle témoigne du danger lui-même, le vital danger de voir ce qui se passait à Birkenau. Elle témoigne de la situation d'urgence, et de la quasi-impossibilité de témoigner à ce moment précis de l'histoire. (35)

Regarder et voir cette image demande que soit considéré ce qui transparaît à sa surface, son contexte ou sa situation. L'image requiert un regard actif, qui pourrait entendre la photographie comme un témoin.

Sous ce même angle, les photos prises par Didi-Huberman en 2010, montrant des sols, des barbelés, des bouleaux, disent en même temps la vie, l'échec de la solution finale, de sa mécanique de mise à mort; elles disent donc quelque chose d'Auschwitz, affirment la vie malgré Auschwitz. Et c'est ainsi que Didi-Huberman se décrit, marchant à travers les vestiges, regardant, se souvenant, réfléchissant, éprouvant des sensations, des émotions : un individu en vie malgré le projet collectif de mise à mort.

Il est évident que le texte d'Écorces tisse très étroitement les thèmes de la trace, de la mémoire, de l'empreinte, s'arrête particulièrement sur la question des sols, souvent négligés et tellement révélateurs ; sur la question de la surface et de ce qu'elle révèle, de ce qui est passé par elle, ce qui a été vécu là où on ne voit plus qu'une face muette :

les philosophes de l'idée pure, les mystiques du Saint des saints ne pensent la surface que comme un maquillage, un mensonge : ce qui cache l'essence vraie des choses. Apparence contre essence ou semblance contre substance, en somme. On peut penser, au contraire, que la substance décrétée au-delà des surfaces n'est qu'un leurre métaphysique. On peut penser que la surface est ce qui tombe des choses : ce qui en vient directement, ce qui s'en détache, ce qui en procède donc. Et qui s'en détache pour venir traîner à notre rencontre, sous notre regard, comme les lambeaux d'une écorce d'arbre. (68)

À Auschwitz-Birkenau, le regard est, comme les pas du visiteur, orienté, balisé, prédéfini. Celui-ci se trouve face à des pancartes, des flèches guidant le regard, des textes écrits disant ce qui 
est à voir. En tant que lieu et événement historiques, Auschwitz est aussi raconté, décrit, enseigné - rassemblant des discours qui structurent le regard. Le regard et ce qui est vu sont indissociables des mots, les camps étant devenus des lieux saturés de langage. S'ajoute le fait que ce qui est donné au regard est configuré en fonction d'exigences culturelles qui ne sont pas neutres : «La culture [...] c'est encore et toujours un lieu de conflits où l'histoire même prend forme et visibilité » (52) - un baraquement converti en stand commercial ; des photos sont recadrées au nom d'une exigence pédagogique de «lisibilité »; des blocks sont transformés en lieux d'exposition : «Ici plus qu'ailleurs les murs mentent : une fois dans le block, je ne peux plus rien voir de ce qu'est un block » (63). Le regard et le visible sont rendus possibles, conditionnés et empêchés par le langage et le statut de lieu de mémoire des camps : «que dire quand Auschwitz doit être oublié dans son lieu même pour se constituer comme un lieu fictif destiné à se souvenir d'Auschwitz ? (67), demande Didi-Huberman - question qui demeure ouverte...

L'exigence de conserver les camps comme lieux de mémoire, d'en faire le support matériel du souvenir, ont produit un recouvrement, un oubli d'Auschwitz devenu invisible : « Auschwitz, aujourd'hui, tend vers le musée » (74), mais l'auteur ne regrette pas ce statut - « c'est tant mieux » (74). Ce statut montre seulement que le visible ne l'est pas immédiatement, ou qu'il est complexe. Il ne s'agit pas, à partir de là, de rejeter le langage, le savoir et la mémoire au nom d'un regard pur, immédiat, simple - rejet aussi absurde que celui a priori des images. Il faudrait plutôt pouvoir s'astreindre à contourner les obstacles du langage et du visible, ceux des conditions de la réalité muséale, pour construire un autre regard, tenter de voir ce qui n'apparaît pas immédiatement - ce qui de la réalité nommée Auschwitz demeure.

\section{Apprendre à regarder : construire un autre regard}

Pour Didi-Huberman, il s'agirait de s'efforcer de «regarder comme regarde un archéologue » (77), selon le modèle de Michel Foucault dans L'archéologie du savoir : ce qui constitue une interrogation des actes de regard ; un «travail du regard » (78). Tout d'abord, tourner le regard vers d'autres objets que ceux que la culture et la muséographie conditionnent à voir, les regarder à un autre rythme. Par exemple, à Birkenau : regarder le sol « qui ne ment pas » (33), ces sols que personne n’a refaits - «[...] brisés, blessés, criblés, fendus. Ces sols entaillés, balafrés, ouverts » (45), portant les signes de la violence qui a eu lieu, subie par des corps massacrés violence dont quelque chose persiste dans ces béances. Ou ce champ, à l'écart, ressemblant à 
n'importe quel autre, alors qu'il est l'emplacement où des fosses ont été creusées pour y jeter des milliers de cadavres, « dans de grands brasiers à ciel ouvert » (53). Les fosses ont été comblées, la végétation a tout recouvert, « il n'y a plus rien à voir de tout cela » - sauf, le long de la clôture électrifiée, une bande rectiligne de fleurs sauvages, un «pullulement bizarre de fleurs blanches sur le lieu exact des fosses de crémation » : «[... on voudrait croire à ce qu'on voit d'abord, à savoir [...] que les morts ne sont plus là. Mais c'est tout le contraire que l'on découvre peu à peu [...]. Ils sont là, ils sont bien là : là dans les fleurs des champs, là dans la sève des bouleaux (67) ».

Quelque chose persiste dans des signes présents, relatifs et fragmentaires, mais exprimant et faisant sentir la mort, le massacre. Ces signes vus seraient des images qui ne sont pas « la chose entière » (35), mais qui montrent pourtant quelque chose. Voir Auschwitz est voir ces signes de «la violence du lieu», et par eux ressentir quelque chose de cette violence, ou éprouver l'impossibilité d'imaginer cette violence. Il y a une expérience du regard, celle de traces par lesquelles quelque chose d'Auschwitz persiste et nous atteint : « J'ai regardé les arbres comme on interroge des témoins muets » (36).

Didi-Huberman prend des photographies lors de sa visite qui ne sont pas des photossouvenirs. Faire des photos est un autre moyen pour un regard archéologique. Étrangement, il retrouve le geste de celui qui, plus de cinquante ans auparavant, avait pris quatre images à la mort, évidemment pas pour les mêmes raisons, même si l'auteur éprouve à faire ses photos « une sorte d'urgence » qui le conduit à les réaliser lui aussi « à l'aveugle » (64). Cette décision est également une exigence : ne pas «transformer ce lieu en une série de paysages bien cadrés » (66), en fonction de normes culturelles, d'un savoir guidant le choix de ce qu'il est important de photographier, d'autres images mille fois vues, mais laisser les images montrer d'elles-mêmes. Le regard est confié aux images qui ne traduisent pas un regard mais sont le regard. Les photographies regardent et ce regard fait voir ce qui n'avait pas été perçu, que l'on n'aurait pas eu l'idée de regarder, ce qui du visible n'est pas d'abord apparu. Elles sont l'occasion d'une sorte de nouveauté du regard et de traversée, par le regard, des couches sédimentaires du visible : «aujourd'hui, en regardant cette image, je m'aperçois de tout autre chose » (71).

Photographier donne aussi la possibilité de comparer son image à d'autres et, par cette comparaison, entre les deux images, quelque chose apparait, devient visible. L'image photographique n'est pas qu'une reproduction superficielle, nécessairement distincte de la réalité, 
elle est un moyen de regarder et de voir, de constituer un autre regard contournant les écrans dressés par le langage, le visible, le regard habituel et habitué.

Les images visuelles, mentales, photographiques seraient comparables à des morceaux d'écorce, des fragments détachés de la réalité. À leur façon et dans leurs limites, elles sont des morceaux de la réalité et font voir ce qui, de celle-ci, peut persister dans l'image. Et les sols détruits de Birkenau sont à leur manière des images, «comme l'écorce de l'histoire » (39). Il ne s'agit pas pour autant de survaloriser l'image par rapport au langage : l'image, comme le langage, peut être le moyen d'un témoignage sans être définie comme l'unique, ni même un moyen supérieur. Le travail de Didi-Huberman sur les images s'accompagne d'une critique et d'une sélection des images, des types d'image, d'une méfiance aussi, l'image pouvant mentir ou rendre aveugle - ni plus ni moins que le langage. Le réel, le discours et le visible sont complexes, hantés les uns par les autres, constitués d'un empilement compact de «sédiments » (58) que les images peuvent contribuer à fouiller pour en extraire des vestiges, des traces, des signes témoignant au présent.

De même que l'écorce n'est pas l'arbre mais dit pourtant quelque chose de l'arbre, les images sont des morceaux d'écorce tombés du réel qui témoignent, partiellement, fragmentairement, de celui-ci. Le réel, par-delà le temps de l'histoire, dure et persiste dans des signes qui parlent. Prendre des photos d'Auschwitz, en produire des images, chercher à voir, c'est continuer à faire parler Auschwitz, témoigner encore de cette réalité inimaginable, si l'on comprend que la mémoire « ne sollicite pas seulement notre capacité à fournir des souvenirs circonstanciés » (30). «De quoi témoignent les photographies d'Auschwitz-Birkenau prises par moi, plus de cinquante ans après la libération des camps ?» (33), demande Didi-Huberman :

Je me suis retrouvé, une fois rentré chez moi, devant ces quelques bouts d'écorce, cette pancarte de bois peint, cette boutique de souvenirs, cet oiseau entre les barbelés [...], cette traînée de fleurs des champs en face du crématoire $\mathrm{V}$, ce lac gorgé de cendres humaines. Quelques images, c'est trois fois rien pour une telle histoire. Mais elles sont à ma mémoire ce que quelques bouts d'écorce sont à un seul tronc d'arbre : des bouts de peau, la chair déjà. (31)

Ce que Didi-Huberman nous invite à lire, dépasse le récit - est au-delà des mots du récit, se cache derrière l'écorce du temps. 


\section{Conclusion}

Ainsi revient-il au montage d'établir des relations entre le présent et le passé, l'image et le vécu dans un souci de connaissance historique ; connaissance qui noue finalement une expérience de l'imagination et un enjeu historique dans un «moment de mémoire » (Benjamin, Fragments, 23) :

La connaissance historique n'advient qu'à partir du «maintenant », c'est-à-dire d'un état de notre expérience présente d'où émerge, parmi l'immense archive des textes, images et témoignages du passé, un moment de mémoire et de lisibilité qui apparaît [...] comme un point critique, un symptôme, un malaise dans la tradition qui, jusqu'alors, offrait au passé un tableau plus ou moins reconnaissable. (Images, 151)

Ce moment de mémoire au cœur de l'expérience présente que Benjamin saisit dans l'étincelle de l'image advient précisément, nous dirions, dans la rencontre d'un effort de l'imagination et d'un effort du montage; d'un effet de surprise issu par la mise en sens, la nomination et l'écriture de la singularité de l'image et du moment historique dont elle témoigne. C'est dire que la mémoire est le résultat d'un montage anachronique ; c'est reconnaître qu'avant d'être travail, elle est émergence immédiate, expérience subjective. Et c'est dans l'actualité de nos regards qu'il s'agit alors de la faire revivre. Car les images et les archives de la mémoire continuent de circuler, de disparaître et de réapparaître, parfois lorsqu'on s'y attend le moins. Ceci pour dire qu'elles ont, même en tant qu'images d'archives, marqué notre imagination, nos rêves, et que nous avons constamment à les activer, comme survivances de la modernité. Il s'agit en somme de poursuivre le travail de montage de mise en relation que ces images impliquent, à la fois pour reconnaître leur présence fantomatique en nous, à côté d'autres images, et pour les laisser s'ouvrir au réel qu'elles désignent encore :

La valeur de connaissance ne saurait être intrinsèque à une seule image, pas plus que l'imagination ne consiste à s'involuer passivement dans une seule image. Il s'agit, au contraire, de mettre le multiple en mouvement, de ne rien isoler, de faire surgir les hiatus et les analogies, les indéterminations et les surdéterminations à l'œuvre. (DidiHuberman, Images, 151)

Le montage suppose déplacement, anticipation, régression. Régression vers des survivances ; régression vers un regard d'enfant sur le monde, soumis à de multiples altérations dans le déplacement possible de l'œil qui contemple le réel et les images immédiates qu'il produit : «Ouvrir le voir, cela signifie prêter attention - une attention qui ne va pas de soi, qui exige un 
travail de pensée, remise en question perpétuelle, problématisation toujours renouvelée aux processus anticipateurs de l'image »(Devant, 220).

Les images de la Shoah nous reviennent et nous les regardons comme des images documentaires rares; elles sont des images-symptômes, elles se déplacent, se fixent, flottent, comme on dit de l'angoisse qu'elle est flottante, sans objet. Mais elles sont aussi déjà prises dans un montage ou à monter, en tout cas engagées dans des modalités discursives qui problématisent leur valeur imaginaire et de connaissance. Cet effort que Didi-Huberman nous incite à développer, entre imagination du possible de l'homme et connaissance par le montage, pourrait nous rendre plus attentifs à la réapparition de ces images devant d'autres et aux retours du terrible dans l'horizon de notre monde contemporain.

\section{Bibliographie}

Benjamin, Walter. Fragments. Tr. C. Jouanlanne et J.-F. Poirier. Paris : Collège international de philosophie/PUF, 2001.

---. «L'œuvre d'art à l'époque de sa reproductibilité technique » [1939]. CEuvres, t. III. Tr. M. de Gandillac, P. Rusch et R. Rochlitz. Paris : Gallimard, 2000.

Didi-Huberman, Georges. Devant le temps. Histoire de l'art et anachronisme des images. Paris : Minuit, 2000.

---. Écorces. Paris : Minuit, 2011.

---. Images malgré tout. Paris : Minuit, 2003.

---. « La muse et le chiffonnier ». Ninfa moderna. Paris : Gallimard (2002) : 122-143.

---. La ressemblance informe ou le gai savoir visuel selon Georges Bataille. Paris : Macula, 1995. Foucault, Michel. L’archéologie du savoir. Paris : Gallimard, 1969.

Kertész, Imre. Être sans destin. Tr. Charles Zaremba et Natalia Zaremba-Huzsvai. Arles : Actes Sud, 1997.

Lanzmann, Claude. Le lièvre de Patagonie. Paris : Gallimard, 2009.

---. Shoah (film). 2003.

Levi, Primo. Si c'était un homme. Tr. Martine Schruoffeneger. Paris : Julliard, 1947.

Rancière, Jacques. «La phrase, l'image, l'histoire ». Le destin des images. Paris : La Fabrique, $2003: 33-51$.

---. « S'il y a de l'irreprésentable ». Le genre humain 36 (2001) : 67-90. 


\begin{abstract}
Notes
${ }^{1}$ Voir : Shoah, film (2003).

${ }^{2}$ Walter Benjamin, «Imagination » (1920-1921) dans Fragments, tr. C. Jouanlanne et J-F. Poirier, Paris, Collège international de philosophie/PUF, 2001, p. 147 : «C'est le propre de l'imagination que d'entraîner les formes dans un jeu de dissolution. Le monde des nouvelles apparitions, ce qui se constitue avec la dissolution de ce qui a été mis en forme, a ses propres lois qui sont celles de l'imagination et dont la loi suprême est que l'imagination, si elle déforme, ne détruit pourtant jamais ».
\end{abstract}

